\title{
Cargo-bicycle with a polymeric frame: The development of a low-cost multifunctional cargo bicycle
}

\author{
Oliver Fonseca ${ }^{1}$, Carlos Relvas ${ }^{2}$ \\ 1EDAM, MITPortugal (201408466@fe.up.pt); 2Department of Mechanical Engineering, \\ University of Aveiro, Aveiro, Portugal (crelvas@ua.pt)
}

\begin{abstract}
Since the beginning of the 21st century, human-powered vehicles are increasingly seen as an attractive alternative to solve some of the problems associated with mobility in major cities. Firmly rooted in Northern Europe's Social and cultural behaviour, cargo bicycles are beginning to spread all over the globe and, in this sense, it is expectable a global outbreak on this market.

A cargo bike that considers the use of a polymer as a raw material to build its frame opens a new window of opportunities for new and disruptive designs. A frame like this raises some new shape and structural challenges. But by taking advantage of existing technologies and combining them, made it possible to achieve results that exceeded by far the initial expectations.

It is not intended to reinvent the bicycle, neither to propose it for new uses, others already addressed these issues. Instead, the goal is to redesign this machine, exploring new frame designs, to adopt Polypropylene as a structural material and explore injection moulding as a manufacturing processes.
\end{abstract}

Subject Headings. Product Design, Sustainable Mobility, Plastics

Author Keywords. Bicycle, Cargo, Utility, Multifunctional, Polymer, Polypropylene

\section{Introduction}

It is noticeable, from the beginning of this century, an increasing interest for mobility solutions alternative to the automobile. According to Wilson (Wilson, 2004) the bicycle should not only be seen as machine but instead, it should also be considered as a driver for social development. Considering this premise, this work only intends to add a small contribution to the technological enhancement of this social developer.

The social changes that are boosting the bicycle demand are increasingly evident in medium and large cities. Even in Portugal, where the auto culture is quite prevalent, there are some signs of acceptance for alternatives to motorized vehicles. This phenomenon becomes more evident when we witness what local and central politicians are implementing in their territories, whether when we witness the increase of bicycle lanes infrastructure or when we witness the recent changes to the traffic and road safety laws. This article is not a sociological study but nevertheless, it is important to spot what is catalyzing these changes to understand how the market is moving and all the businesses that are arising from this social ecosystem.

Since the beginning of the 21st century's second decade, the Portuguese bicycle market started to show some signs of activity. Furthermore, initiatives started to sprout, being the main promoters, of these new initiatives, organizations like the: Portuguese Federation of Cycle Tourism and Bicycle Users (FPCUB, 2014), the "Lisboa Ciclável" platform promoted by the Lisbon Council (CML, 2014), the Portuguese branch of the movement "Massa Crítica" (Anon., 2014), associations like MUBI (MUBI, 2014), the "de Bike para o Trabalho" (Bike Anjo, 2014 ) initiative, and also, the cycle of congresses for soft mobility promoted by ABIMOTA LEA 
(ABIMOTA LEA, 2013). Despite this efforts, things are still somehow incipient when compared with other initiatives worldwide.

In the US there are some strong bicycle lobby groups such as the "Alliance for Biking and Walking" (AB\&W, 2014), "People for Bikes" (peopleforbikes.org, 2014), "CyclingSavvy" (Cycling SavvY, 2014), "International Mountain Biking Association" (IMBA, 2014), "League of American Bicyclists" (TLAB, 2014), "National Bicycle Greenway" (NBG, 2014), "Rails-to-Trails Conservancy" (Rails To Trails, 2014), "Adventure Cycling Association" (ACA, 2014). The latter are usually called Bicycle Advocates and in total there are roughly 77 local and state associations in the US. There are also major private organizations in the sector as is the case of the 2-million-dollar fund owned by SRAM (SRAM, 2014) that supports initiatives to improve and build infrastructures that promote the use of bicycles. Paradoxically the United States is one of the countries with the lowest rates of bicycle usage, averaging no more than $1 \%$ of the total trips (IBF, 2014).

In North European states like Holland, Denmark and Sweden, this reality is completely different. These countries are taking the lead in alternative mobility solutions. This reality is not only driven by historical and cultural reasons but are mainly related with a need to manage efficiently traffic congestion inside the cities.

"The Netherlands, Denmark, Germany, and other European nations reached high cycling rates through policies that give priority to cycling, walking, and public transportation over private automobiles". (Worldwatch Institute, 2013)

"...ample bicycle parking, and coordination with public transport have all made cycling safe, fast, and convenient in strong biking cities." (Worldwatch Institute, 2013)

Also in Central Europe, some cities, in countries like Germany and Switzerland, start to emerge as being bike friendly. Two good examples of these cities, changing for this bicycle proliferation, are the cities of Munich and Stuttgart (Petty, 2001). In these cities the bicycle is increasingly considered as the perfect countermeasure for pollution and traffic jams in the city center.

After identifying these signs of an emerging bicycle culture, it was found challenging to give a modest contribute to this movement. In this sense, the way found to fulfill this was to design and develop a practical, urban-oriented, affordable, light weighted and compact cargobicycle. Furthermore, the developed bicycle is intended to address a wide range of consumers, i.e. a mass produced product oriented to the global market.

\section{Project's Methodology}

In order to structure the creative thinking, and to narrow the scope of actions on the outlined objectives, a methodology was followed. The method that guided this work throughout the process of product design and its subsequent development is mostly inspired on the work of Ulrich and Eppinger (Ulrich \& Eppinger, 2012). However, it is relevant to refer that this project was not entirely constrained to the above referred method. This option created space to use outer tools to adjust the original method to the project needs. Hence, the work followed these basic steps:

\subsection{Identifying costumers and their needs}

To identify customer needs, it was necessary to determine who these customers were, and in which regions of the world they were. Determining the product's state of the art also helped to determine where the main target markets were. This kind of information is vital because it 
allows to identify specific cultural requirements. Estimate the market size and doing a competitive benchmark, were the next obvious steps in assessing the opportunity and spot the competitor's strengths and weaknesses.

\subsection{Defining product specifications}

Specifications were defined in the pre-conceptual phase. Here It was possible to determine, from previous analysis, a set of competitive attributes and specifications. These were considered and included in the conceptual proposal. Using the Kano model it was possible to determine and classify the necessary attributes to fulfill each specification. On a later stage this information was analyzed in a Quality Functional Deployment (QFD), qualitatively and quantitatively, to hierarchize all the specifications.

\subsection{Concept generation}

The existing set of a key features originated the first conceptual ideas, triggering the sketching process. These freehand sketches were fundamental to start translating ideas into concepts. At first, these drawings disregarded any information collected previously, in an attempt of creating the largest number of concepts without the contamination of preconceived ideas. In a second phase, the work leaped forward with the integration of technical details and other key features. Finally, during the third phase, the computer aided design (CAD) stood out, and at this stage, all selected requirements were developed. In addition, anthropometric and ergonomic parameters were also included.

\subsection{Concept selection}

At this point, it became necessary to set a course of action and define a final design for a consensual proposal. This could be one of the developed proposals or a completely new one resulting from the merge of existing ideas. The set of developed concepts was tested using failure mode and effect analysis (FMEA). This procedure highlighted the best ideas and alerted to potential failures. From this, in the end, it was possible to merge the best ideas in a single concept.

\subsection{Concept testing}

The main concern at this stage, was to give an assertive answer to uncertainty in critical components developed for the digital prototype. In these case the prime tool to deal with this uncertainty was finite element analysis through computer aided engineering (CAE).

\subsection{Defining the final specifications}

From this point on, the product was developed in a manufacturing perspective. So, it became a priority to plan and prepare all the support information and the technical documentation necessary to manufacture the product. These activities were focused in the optimization of the processes of Design for manufacturing an assembly (DFMA).

\section{Market size}

Figure 1 presents data collected from prominent industrial and commerce associations. The first fact that immediately emerges from the figure is the dimension of the Chinese manufacturing industry (BIKEeurope, 2012), but this does not mean that China is alone in the bicycle manufacturing business. Examples of other big players are India with 15 millions of units produced in 2001 (AICMA, 2002), the European Union (EU) with 11 milions of bicycles manufactured in 2012 (COLIPED, 2013), Brazil with 4 millions produced in 2012 (ABRACICLO, 2013), 2 millions produced in Japan in 2008 (IBF, 2014), and within the United States (USA) a retail market that sold 16 million bicycles in 2013 (NBDA, 2014). 


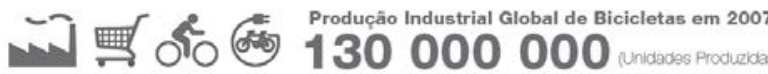

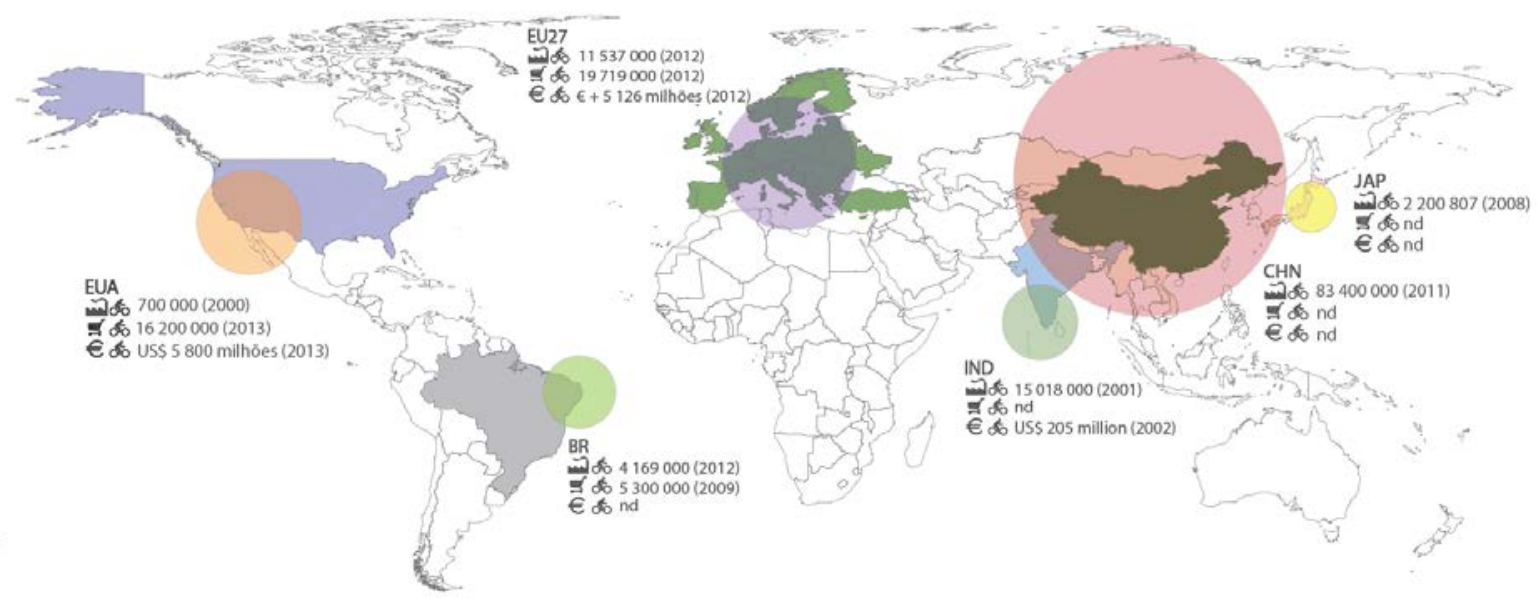

Figure 1: Bicycle's global market (Fonseca, 2014)

\section{The Design and development processes}

The challenge, from a design point of view, was to answer to four major issues that were: "What?", "Why?", "Who?" And "How?". The answer to these questions is often subjective, however, it is of vital importance to justify the relevance of the project. The answer to the first question is answered by the identification of the opportunity, while the remaining are answered during the course of the project. The answer to the question "How?" occupies a lead role throughout the entire project, resulting on a continuous questioning process that conducted, in the end, the work to a feasible proposal.

\subsection{Goals and requirements}

The main objective was to design a bicycle's frame which was: multifunctional, lightweight, fast to manufacture and with a low overall coast. In short the product should accomplish the following objectives:

\subsubsection{Bicycle's general functional requirements}

- Must be compact,

- Must steer well in tight places,

- Must allow customization through specific add-on's,

- Must allow the installation of premium add-on's,

- Must be suited to carry infants,

- Must suit people with heights from 1,5m to 1,95m,

- Must carry 1 cubic meter objects in a front rack,

- Must have a gear shifting system (variable transmission).

\subsubsection{Bicycle's frame mechanical performance requirements}

- Must resist to a load greater than $3000 \mathrm{~N}$ at the seat pool,

- Must resist to a load greater than $5000 \mathrm{~N}$ at the steering bushing,

- When the loads are applied, the displacement must be lower than $2 \mathrm{~mm}$,

- For calculus simplification, the frame should be considered fixed at the pedal shaft housing. 


\subsubsection{Bicycle's frame Manufacturing requirements}

The product must propose new and leaner manufacturing methods. The approach must consider a mass production scenario in order to achieve the lowest overall price.

\subsection{Materials selection}

The process of materials selecting started at an early stage, and in parallel with the development of the virtual model in CAD. The possibility of using a thermoplastic polymer to materialize the structural elements emerged in early in modeling phase. At first it looked unfeasible, but after some thought what seemed to be a silly idea started to reveal itself as a solution to achieve a more organically shaped frame.

The preliminary analysis to the pool of polymeric materials allowed to narrow down the focus to a small selection of potential candidates. The criteria to achieve this small group of materials was to filter the materials by their transformation process, young's module, yield strength, density and bulk cost. The selection was conducted using, in CES EduPack 2011 (Granta Design Limited, 2010) that is a material selection's dedicated database. The constraints used as filters in this selection process were:

- The material must allow to be processed by injection molding,

- The material's price must not be higher than $3 €$ for kilogram,

- The material must have a good ratio between density and Young's module.

These constraints narrowed the group of thermoplastics to those that fitted the desired profile. Table 1, sums up the materials that passed this filter, and they are the $30 \%$ glass fiber reinforced Polypropylene (PP), the $40 \%$ glass fiber reinforced Acrylonitrile butadiene styrene (ABS) and the $50 \%$ glass fiber reinforced Polypropylene (PP).

\begin{tabular}{|c|c|c|c|c|}
\hline & $\begin{array}{c}\text { PP } \\
\text { (copolymer, impact, } \\
\text { 30\% glass fiber) }\end{array}$ & $\begin{array}{c}\text { ABS } \\
\text { (40\% long glass fiber, } \\
\text { injection molding) }\end{array}$ & $\begin{array}{c}\text { PP } \\
\text { (50\% long glass fiber) }\end{array}$ & \\
\hline Density & $1110-1130$ & $1350-1370$ & $1320-1340$ & $\mathrm{~kg} / \mathrm{m}^{3}$ \\
\hline Price & $2.21-2.43$ & $2.79-3.07$ & $2.5-2.75$ & $€ / \mathrm{kg}$ \\
\hline Young's modulus & $3.27-5.12$ & $6.73-7.07$ & $10.4-11.7$ & GPa \\
\hline Compressive modulus & $3.27-5.12$ & $6.73-7.07$ & $10.4-11.7$ & $\mathrm{GPa}$ \\
\hline Flexural modulus & $1.47-1.51$ & $7.38-7.75$ & $10.1-11.2$ & $\mathrm{GPa}$ \\
\hline Shear modulus & $3.98-4.39$ & $2.46-2.59$ & $4.05-4.15$ & GPa \\
\hline Bulk modulus & $5.18-5.31$ & $8.44-8.87$ & $12-12.3$ & GPa \\
\hline Poisson's ratio & $0.366-0.374$ & $0.36-0.374$ & $0.345-0.352$ & \\
\hline Shape factor & 13.3 & 6.9 & 14.3 & \\
\hline Yield strength & $53.3-67$ & $84-92.8$ & $110-129$ & $\mathrm{MPa}$ \\
\hline Tensile strength & $47.9-59.3$ & $105-116$ & $115-127$ & $\mathrm{MPa}$ \\
\hline Compressive strength & $52.5-55.1$ & $112-123$ & $80.7-126$ & $\mathrm{MPa}$ \\
\hline Flexural strength & $72.3-93$ & $164-181$ & $177-199$ & $\mathrm{MPa}$ \\
\hline Shear strength & $x$ & $\mathrm{x}$ & $x$ & $\mathrm{MPa}$ \\
\hline Elongation & $4.81-7.73$ & $1.4-1.61$ & $1.53-1.85$ & $\%$ \\
\hline Elongation at yield & $4.23-5.29$ & $x$ & $1.27-2$ & $\%$ \\
\hline Hardness - Vickers & $17.5-18.4$ & $25.2-27.8$ & $34.9-36.7$ & HV \\
\hline Fatigue strength @10^7 cycles & $20.8-21.8$ & $38.8-50.4$ & $47.1-49.5$ & $\mathrm{MPa}$ \\
\hline Fracture toughness & $2.75-2.89$ & $4.23-5.08$ & $5.11-5.37$ & MPa.m $\mathrm{m}^{0.5}$ \\
\hline Mechanical loss coefficient & $0.0146-0.0153$ & $0.0102-0.0105$ & $0.00726-0.00762$ & \\
\hline Embodied energy & $93.1-103$ & $95-105$ & $97.2-107$ & $\mathrm{MJ} / \mathrm{kg}$ \\
\hline CO2 footprint & $6.24-6.89$ & $6.4-7.08$ & $6.58-7.27$ & $\mathrm{~kg} / \mathrm{kg}$ \\
\hline Polymer molding energy & $20.2-22.3$ & $16.8-18.6$ & $18.7-20.7$ & $\mathrm{MJ} / \mathrm{kg}$ \\
\hline Polymer molding CO2 & $1.51-1.67$ & $1.26-1.39$ & $1.41-1.55$ & kg/kg \\
\hline Recycle & No & No & No & \\
\hline
\end{tabular}

Table 1: Materials and manufacturing process for the bicycle's frame (Fonseca, 2014) 
From this group, PP charged with $50 \%$ long glass fibers, arises as the most promising material not only because it had the highest Young's module, but also because it had the highest Yield Strength. Furthermore, the availability and relative ease to use this material in a manufacturing process strengthened the decision.

\subsection{Model generation and Model Validation}

This phase follows the process of developing ideas and concepts. Here the ideas and requirements are developed and interconnected, in order to virtually prototype the product. From hereon, parts final morphology, features and technologies are embodied in the virtual model and the concept starts its transformation process to a product that meets the outlined goals.

Figure 2 presents a rendered image of the basic proposal. The rendered image (Figure 2) illustrates a bicycle witch already incorporates an add-on in this case a transporter box, showing one of the multiple add-on choices for the proposal. The base product may, in essence, be reduced to its structural elements. But also these, in the end, may accommodate different options and add-on's. This results from an effort to modularize the product's architecture allowing to assemble a wide range of solutions. However, at this point, to simplify the product's bill of materials (BOM) management, a base or standardized proposal will be presented, which corresponds to the expectations of a majority of potential buyers. Nevertheless, for the more demanding, some variant proposals are also under development.

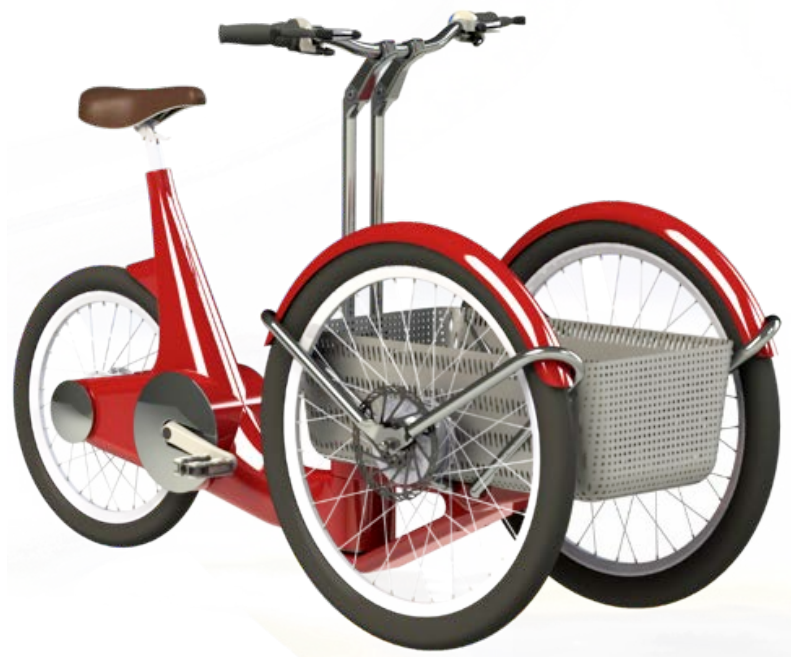

Figure 2: 3D Render of the proposal (Fonseca, 2014)

At a first glance, developing a new bicycle may appear to raise the question of taking into account very seriously the ergonomic and anthropometric concepts, however, these concepts, in reality, usually emerge as limitations. When trying to adjust a product to a universe of individuals, were each one has his unique anthropometric characteristics, a good solution for one individual can be completely misfit to the rest of the population. Also, the refinement of a particular part or system to the specific needs of one individual, may also critically jeopardize its function or performance.

For this, Burrows (Burrows, 2008) argues that full ergonomic performance in a bicycle is not a tangible feature, he even claims that ergonomics on bicycles cannot be seen as an exact science, but rather as a set of criteria, more or less empirical, mostly connected to personal tastes and anatomic characteristics of each individual. Therefore, in this project all decisions in the field of ergonomics, were carefully weighed and assessed to determine their added value. One of the most important decisions, with impact in comfort, is the posture that the 
cyclist takes on the saddle. This position is mostly influenced by the angle of the handlebar and the height of the seat relating to the ground. To manage this the proposal, like many other bicycles, includes a height adjusting system that allows the rider to adjust the seat position and handlebar stem angle according height to his personal preference (Figure 3). That approach demonstrated to be the most consensual way to adjust the posture of a rider.
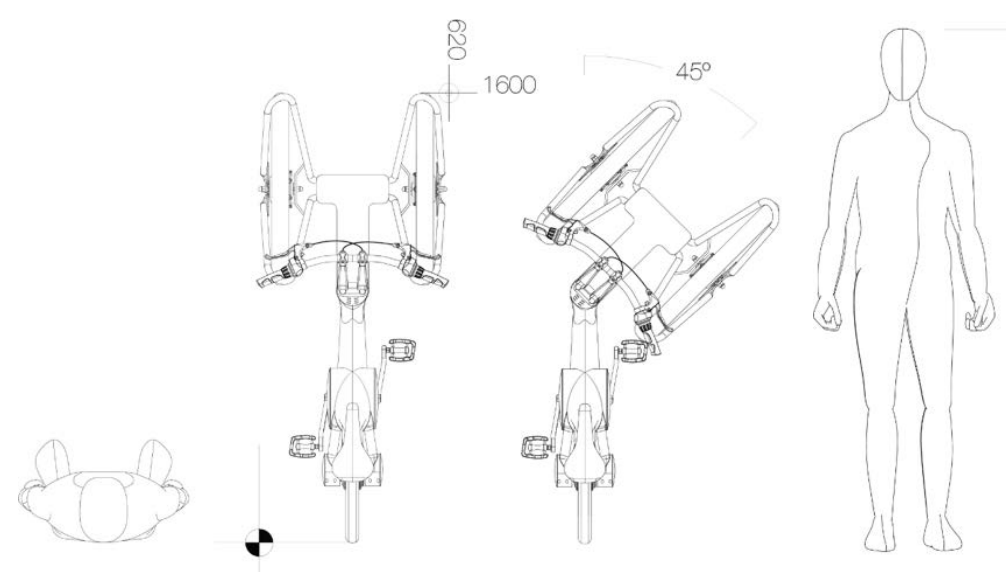

$-1800$

Figure 3: Proportions of the proposed bicycle, comparing with human body (Fonseca, 2014)

After modeling ideas by a process of shape refinement, and after selecting the materials, it became not only necessary to theoretically validate the structural performance of the model, but also to evaluate its technical feasibility and manufacturability as well. This assessment through finite element analysis with "SolidWorks" CAE suite, allowed to refine all structural elements, by virtually experimenting with them various structural approaches.

This analysis was essential to validate all new parts. Still, the frame's outer shell was the most time consuming element in this analysis, demanding 17 trials that took approximately 8 hours each. But because it was the most important element in the bicycle's structure and at the same time, due to its structural design and the nature of the proposed materials, was the part that introduced more uncertainty in the project's success.

The optimization process involved a systematic and evolutionary analysis. In the case of the frame, the approach to the problem was: in a first phase to learn which were the shape's natural behaviors to a given set of loads, i.e. assigning small loads of $100 \mathrm{~N}$ to a $3 \mathrm{~mm}$ thick shell without any reinforcement elements. Thus, it was possible to perceive and collect important information that pointed out one by one the weak spots in the shell.

From this data, it was possible to start a strategy of systematic testing followed by the introduction of geometric countermeasures that counteracted the undesirable displacements caused by the applied loads. In turn, also the loads applied to the part became increasingly higher to the point in which the loads applied were clearly oversized, i.e. the safety factor was considered to be overtaken. At the last trial (Figure 4), the element was tested with a set of two $3000 \mathrm{~N}$ remote loads applied to the front bushing, and a single load on the seat shim area with $5000 \mathrm{~N}$. These loads produced a maximum displacement of $1.8 \mathrm{~mm}$. 


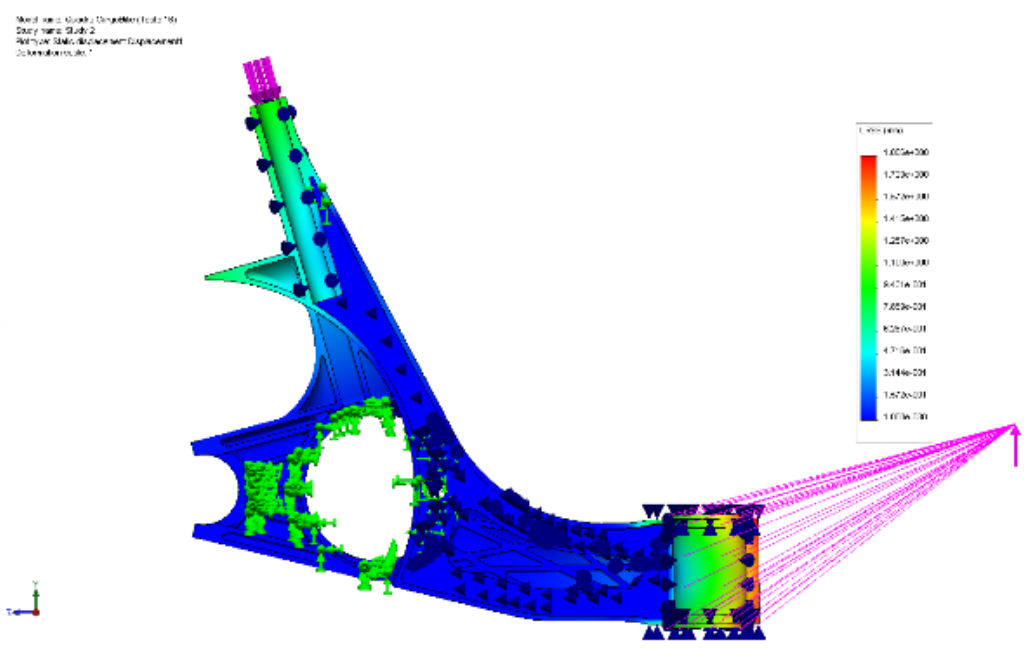

Figure 4: Loads applied to the proposed bicycle frame (Fonseca, 2014)

The assembled frame (Figure 5), detailed in Table 2, is a sandwich of smaller parts assembled in such way that the overall solution is robust and weighs less than $3.5 \mathrm{~kg}$. The frame design focused on a set of materials with different properties. This strategy aimed to produce a structural assembly in which the strength and stiffness of each component alone would not be able to resist to the applied loads, yet all parts combined will perform mechanically as a structure that effectively resist to the typical demands of a heavy duty bicycle.

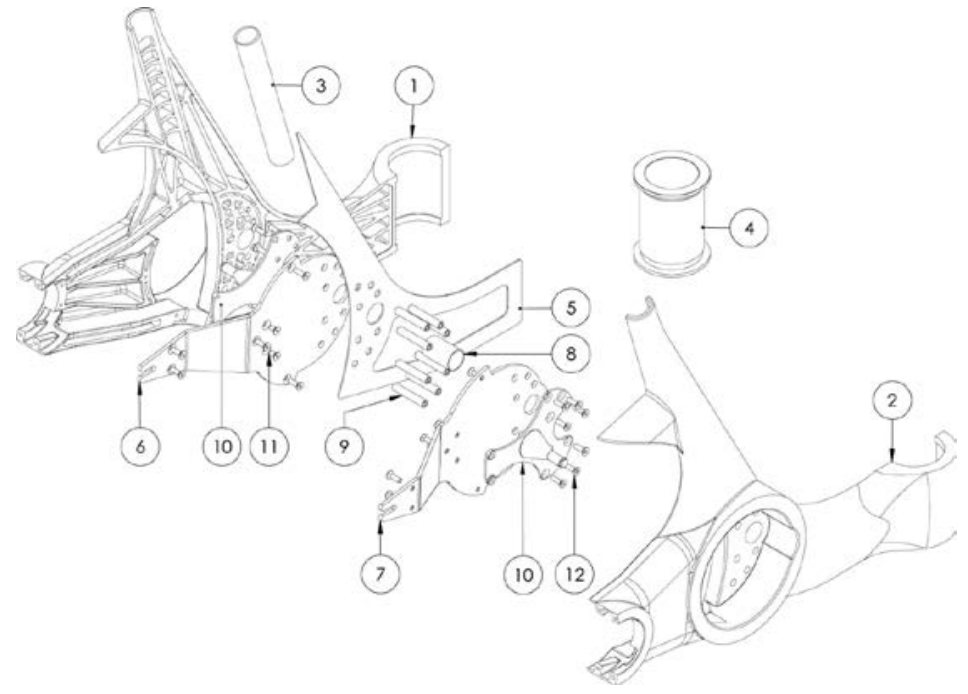

Figure 5: Bicycle's frame exploded view (Fonseca, 2014)

\begin{tabular}{c|l|c|l}
\multicolumn{1}{l|}{ Item } & Part & Material & Process \\
\hline (1)(2) & Frame's outer shell & PP & Injection moulding \\
\hline (3) & Seat pool housing & EN AW-5083 & Extrusion \\
\hline (4) & Steering bearing housing & EN AW-5083 & Injection cast \\
\hline (5) & Central reinforcement blade & AISI304 & LASER cut \\
\cline { 1 - 1 }$(6)(7)$ & Back Wheel brackets & LASER cut + Bending \\
\hline (8) & Shim for offset shaft & EN 36NiCrMo16 & \multirow{2}{*}{ Cut + Machining } \\
\hline (9) & Spacers & DIN CK45k & \\
\hline (10) & Transmission plate and pivot & EN 36NiCrMo16 & Die cast + Machining \\
\hline (11) & Countersunk fastener DIN7991 M6x16 & \multirow{2}{*}{ AISI304 A2-70 } & Standard component \\
\hline (12) & Countersunk fastener DIN7991 M6x30 & &
\end{tabular}

Table 2: Parts, materials and manufacturing process for the bicycle's frame (Fonseca, 2014) 
Similarly, to the strength and stiffness validation there was also the need to validate the parts from a manufacturing point of view. It was perceived, from the developed solution for the frame, that a polymer injection process was a viable process to obtain the external parts. In this sense, the polymer injection process was also simulated, this time using Autodesk inventor CAE for plastics (Figure 5). At start, the strategy for the mould flow testing was similar to the one adopted for the structural validation process. Yet, in this case it was possible to conclude early in the process the component's feasibility, from the feedback of two trials, that tested alternative injection strategies.
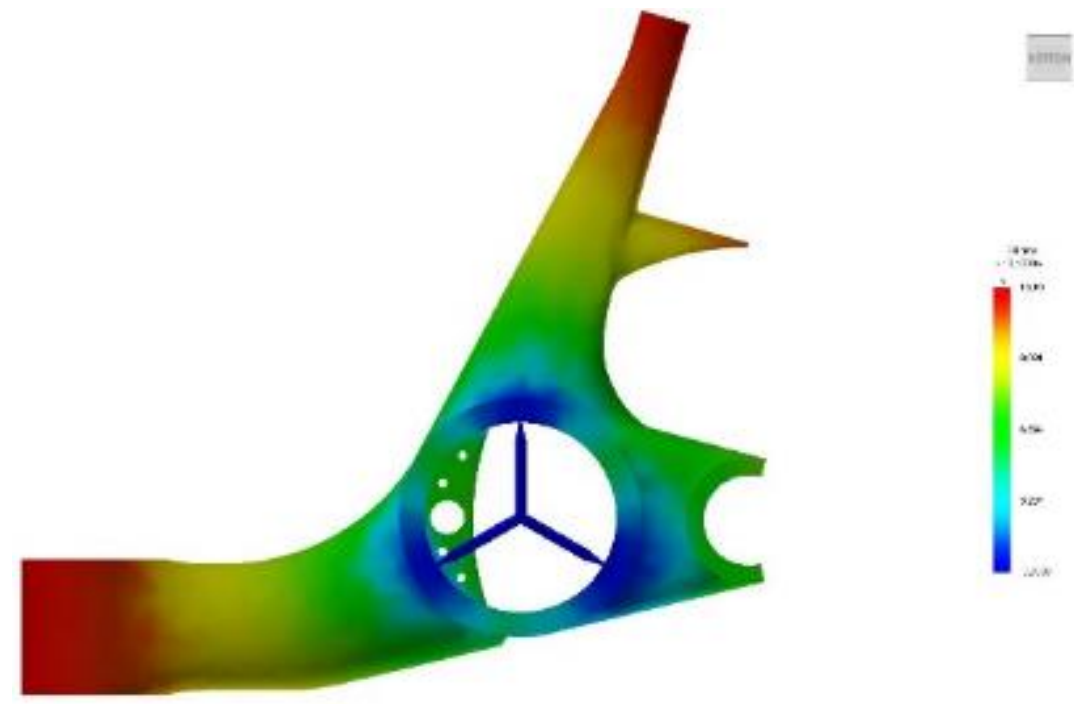

Figure 6: Mould flow for the proposed bicycle frame (Fonseca, 2014)

From all this development and validation processes, the resulting frame proposal consists, fundamentally, on a three parts assembly. The outer shells are internally reinforced with a trussed structure (Figure 6) and concealed inside is a metallic reinforcement that is the third part. The bonding of the parts should be obtained either by ultrasound welding or by using polymeric resin as adhesive. By proposing such an approach to achieve an organic shape and production's time cut, this bicycle's project must be able to compete with the existing solutions.

\section{Systems}

In a strategy of guaranteeing the maximum reliability to the entire product, the selection of standard components and long proven reliable systems was essential to achieve this goal, however this criterion is not confined to the solutions proposed by the bicycle industry. There are, at least, two new systems in this product that have adopted principles and proven systems for other industries.

The first case of this technological migration is the turning bearing, which was replaced by a self-lubricated polymer shim, this solution is widely used in both the automotive industry and in industrial machinery.

The second case refers to the solution adopted to solve the problem of clearance between the rear wheel and the crankshaft. The new bicycle model drastically reduced its total length. Nevertheless, it was necessary to completely redesign the crankset and crankshaft systems. Traditionally this problem is solved in a very simple and reliable way, by installing a shaft that acts as a common axis between the two pedal cranks but in this new frame design this solution was no longer available, because the crankshaft would conflict with the rear wheel. The 
solution was to transfer this common axis to a more favorable position through a spur gears assembly using a 1 to 1 transmission ratio (Figure 7).

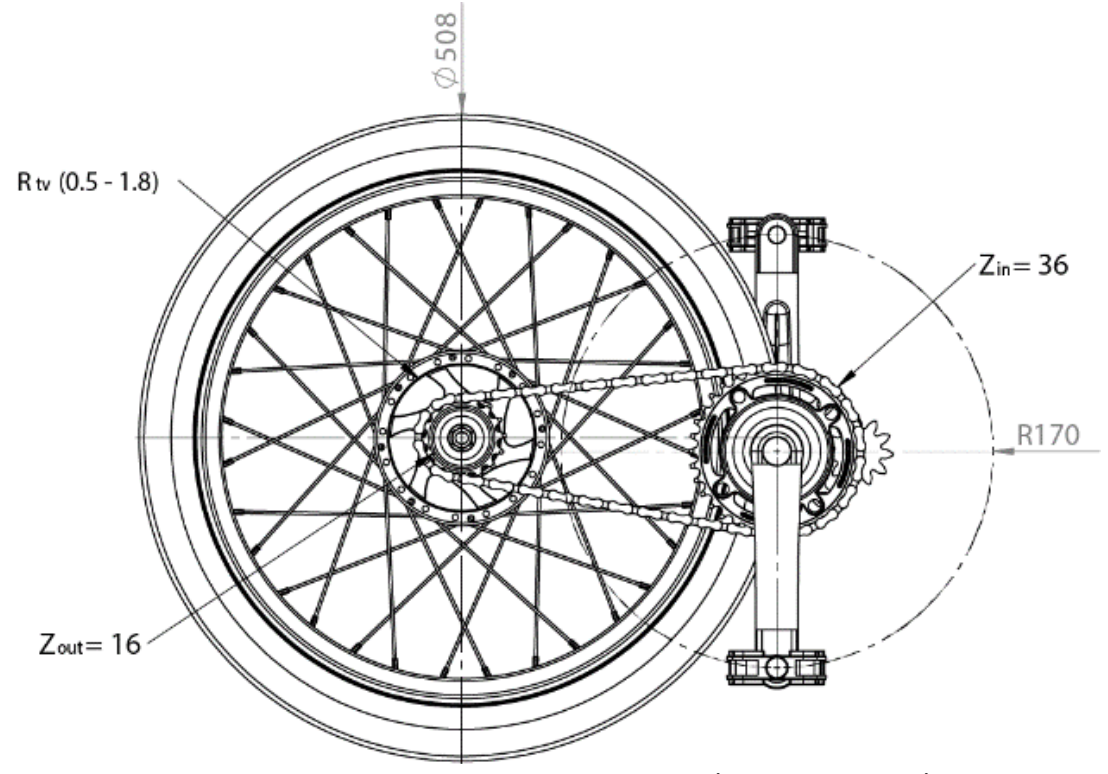

Figure 7: Power transmission system (Fonseca, 2014)

\section{Prototype}

On the final stage of the project, it became imperative to physically prototype the virtual model. The importance of this materialization process is unquestionable because it allowed to evaluate all technical choices like: morphology, aesthetics, structural design and function. Ultimately validating the materialized concept as a product from both design and engineering scopes.

Prototyping is not an inexpensive activity, and to some extent, difficult to justify to some decisions making stakeholders. Nevertheless, one should have in mind that failure in advanced stages of a project are highly costly and have enormous impact in the final budget. Preventing failure in the early stages of the development process, by continuously testing and assessing solutions significantly lowers the coasts due to failure. Obviously, in this particular case, the need for prototyping is limited to the newly developed parts, it would be pointless to prototype shop shelve parts. Therefore, the prototyped parts were subjected to a cost / benefit analysis.

In this project, by only partially prototyping the proposal a practical decision was made. In practical terms it was decided not to fabricate any component that could be replaced from a standard component already existing in the market.

For this prototype the following objectives are succinctly pointed out:

- Evaluate the coherency of the proposal's aesthetics at its real size;

- Evaluate the proportions of the different structural elements;

- Evaluate the relationship between the proposal and the human body;

- Evaluate the relationship between systems and structural parts;

- Communicate the value proposition.

As expcted, the dimensional properties of the prototyped parts, prevented these from being materialized at once or at least in an accurate and cost-controlled way. These difficulties are very evident if we take into account the dimensional limitations of most, affordable, rapid prototyping systems. To overcome this barrier, it was adopted a prototyping strategy that consisted in sectioning the prototyped parts in small sized blocks of 90x90x90mm (Figure 8). 
For instance, the printing process of the right structural half of the frame represented on Figure 8, was accomplished by sectioning this part in 27 block elements and printing during 94 hours. In this particular part the materialization of these 27 elements consumed $1.6 \mathrm{~kg}$ of Polylactic acid (PLA) wire, confirming the 3D model's forecasts that estimated, for this part, a weight below $2 \mathrm{~kg}$.

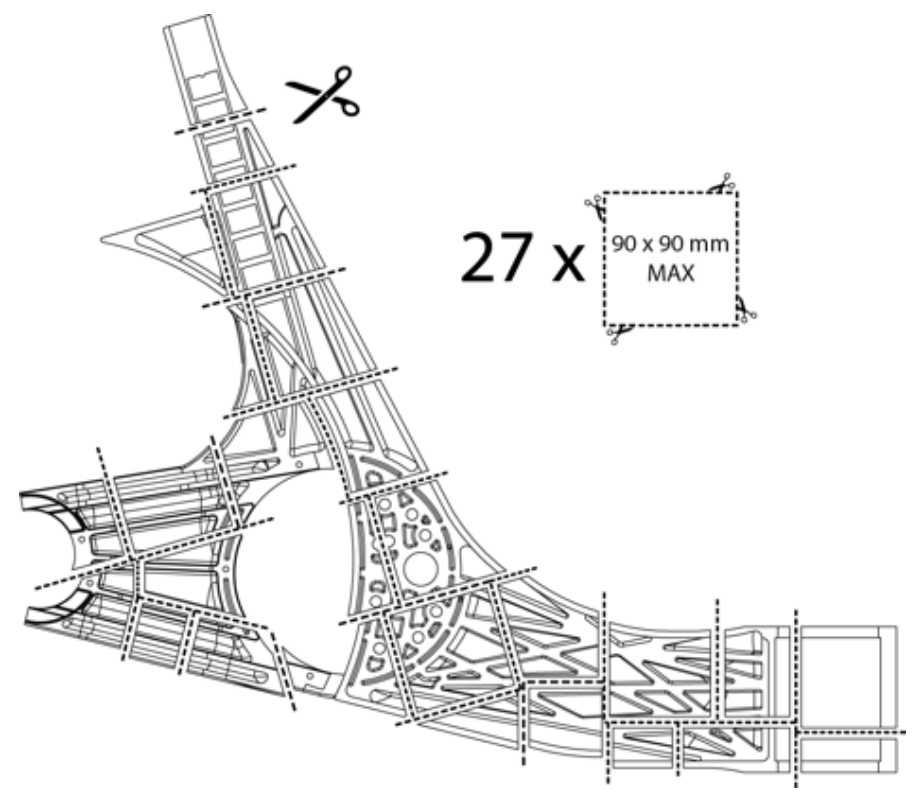

Figure 8: Part sectioning for 3D printing (Fonseca, 2014)

\section{Conclusions}

This research used information from all available media, from academic articles, conference publications, reference books, the "web", reports from associations and industry, specialized magazines and, occasionally, even articles from the mass media. This research took place mainly during the pre-conceptual and conceptual stages and was responsible for the foundations of the project. Nevertheless, often along the project even on advanced stages, new inputs from outside were continuously forcing to question the meaning of the ongoing work.

The analysis of all sources of evidence also proved to be vital in the search for the design. From an early stage, the project's goal was to propose a product alternative to the existing in the market. Meanwhile, there was a clear concern in reaching a compromise between design, function and manufacturability. The systems selection process favoured reliable systems and long proven solutions. This principle was used even in the new solutions, only selecting parts and mechanical systems that proved to be reliable in applications with similar operating conditions.

Assuming an "every day" use bicycle, it was necessary to predict all the tasks that it would be destined for. Therefore, after selecting a viable concept, it became possible to start virtually prototyping with computer aided design tools (CAD). A selected set of early freehand sketches inspired the birth of the model in the new digital medium, resulting in the first virtual model. This embryonic model was subjected to critical analysis for early failure detection and evaluated its convergence with the proposed objectives.

Computer aided design (CAD), allowed to systematically test the incoming ideas. Furthermore, it was possible to implement and design the various systems necessary for the bicycle to run. Ultimately, shape and aesthetics were the characteristics most influenced by this continuous refinement. This quest aimed for a very fine tuned balance between shape, structural 
function, materials and manufacturing process. This criterions refinement of the technological solutions for the structural elements and their manufacturing processes prevented the misrepresentation of the initial concept.

When a structural part is proposed with an organic shape, employing alternative materials and different manufacturing processes, it becomes imperative to theoretically validate this solution. Computational finite element analysis (FEA), is a common tool in theoretical validation process. It plays a major role in design and engineering problem solving. As the designer develops new shapes he can immediately test and optimize them and this way releasing material and shape's full potential.

It would be too risky to propose a new product without theoretically validating its industrial feasibility. This way, now on a manufacturing scope, computer aided engineering tools (CAE) were also used in key components to simulate and analyse their manufacturing process.

This design, resulted in a cargo bike proposal, within the scope of the initially objectives. By proposing a basic and inexpensive model and developing a range of add-ons and accessories that satisfy the individual needs of the diverse users. The customization potential is the differentiation factor from competing products. Through a modular architecture, designed to receive add-ons and premium parts it is intended to produce a wide set of solutions that satisfies a broader number of needs and niche markets. It is through this diversity of solutions that the product aims to reach its global markets.

It is not accurate to state that only the technological developments in materials and manufacturing processes will influence the future of bicycles. Instead, an educated view should also consider political decisions and sociological aspects as drivers for bicycle use. In a near future, bicycles might even find their natural partners in other transportation systems such as buses and trains, competing this way, finally, side by side with cars.

\section{References}

"Massa Crítica * Portugal." 2014. Accessed May 5, 2014. http://www.massacriticapt.net/.

AB\&W (Alliance for Biking \& Walking). 2014. "Alliance for Biking \& Walking - Building the People Powered Movement". Accessed January 22, 2014. http://www.bikewalkalliance.org/.

Abimota Lea. 2013. "Congresso Internacional de Promoção da Mobilidade Suave". Accessed October 21, 2013. http://www.abimota.org/en/a-associacao/eventos/130-congressointernacional-de-promocao-da-mobilidade-suave.

Abraciclo. 2013. "Dados do Setor". Accessed May 24, 2014. http://www.abraciclo.com.br/dados-do-setor.

ACA (Adventure Cycling Association). 2014. "Adventure Cycling Association - America's Bicycle Travel Experts". Accessed February 8, 2014. https://www.adventurecycling.org/.

AICMA (All India Cycle Manufacturers Association). 2002. "Indian Bicycle Industry". Accessed May 18, 2014. http://www.aicma.org/pages/indianbicycleindustry.htm.

Bike Anjo. 2014. "De bike ao trabalho". Accessed April 2, 2014. http://debikeaotrabalho.org/.

Bike Europe. 2012. "China's bike production and export leveling off". Accessed May 2, 2014. http://www.bike-eu.com/sales-trends/nieuws/2012/5/chinas-bike-production-andexport-leveling-off-10110043.

Burrows, Mike. 2008. Bicycle Design. London: Snowbooks, Ltd.

Câmara Municipal de Lisboa. 2014. Lisboa Ciclável. Map. Accessed April 2, 2014. http://lisboaciclavel.cm-lisboa.pt/. 
COLIPED. 2013. "Facts \& Figures (European figures)". Accessed May 11, 2014. http://www.coliped.com/.

Cycling Savvy. 2014. "Cycling Savvy - Empowerment for Unlimited Travel". Accessed January 15, 2014. https://register.cyclingsavvy.org/home.

Fonseca, Oliver Michael Pinto. 2014. "O desenvolvimento de uma bicicleta multifuncional". Dissertação de Mestrado, Departamento de Comunicação e Arte e Departamento de Engenharia Mecânica, Universidade de Aveiro.

FPCUB (Federação Portuguesa de Cicloturismo e Utilizadores de Bicicleta). 2014. "Federação Portuguesa de Cicloturismo e Utilizadores de Bicicleta". Accessed May 25, 2014. http://www.fpcub.pt.

Granta Design Limited. 2010. CES EduPack 2011 [computer program]. http://www.grantadesign.com/education/edupack/edupack2011.htm.

IBF (International Bicycle Fund). 2014. "Bicycle statistical data: usage, productions and sales". Accessed May 20, 2014. http://www.ibike.org/library/statistics-data.htm.

IMBA (International Mountain Bicycling Association). 2014. "International Mountain Bicycling Association". Accessed January 15, 2014. https://www.imba.com/.

MUBi (Associação para a mobilidade urbana em bicicleta). 2014. "MUBi | Associação para a mobilidade urbana em bicicleta". Accessed March 14, 2014. http://mubi.pt/.

NBDA (National Bicycle Dealers Association). 2014. "Industry overview 2014: a look at the bicycle industry's vital statistics". Accessed May 12, 2014. http://nbda.com/articles/industry-overview-2013-pg34.htm.

NBG (National Bicycle Greenway). 2014. "Connecting cyclists to cities - Building a consciousness for the NBG since 1987". Accessed January 19, 2014. http://nbg.bikeroute.com/.

PeopleForBikes. 2014. "PeopleForBikes". Accessed January 15, 2014. http://www.peopleforbikes.org/.

Petty, Ross D. 2001. "The product life cycle and the use of bicycles to deliver goods and services." In Milestones in Marketing History: Proceedings of the 10th Conference in Historical Analysis and Research in Marketing2001, 117-127.

Rails to Trails Conservancy. 2014. "Rails-to-Trails - Inspiring movement creating a nationwide network of trails". Accessed January 16, 2014. http://www.railstotrails.org/.

SRAM Cycling Fund. 2014. "SRAM Cycling Fund". Accessed December 18, 2013. http://www.sramcyclingfund.com/.

TLAB (The League of American Bicyclists). 2014. "The League of American Bicyclists". Accessed January 18, 2014. http://bikeleague.org/.

Ulrich, Karl T., and Steven D. Eppinger. 2012. Product design and development. 5th ed. New York: McGraw-Hill Companies.

Wilson, D. G. 2004. Bicycling Science, with contributions by Jim Papadopoulos. 3rd ed: Cambridge, MA: MIT Press.

Worldwatch Institute. 2013. "Bicycle Production Reaches 130 Million Units". Accessed April 12, 2014. http://www.worldwatch.org/node/5462. 